\title{
Fast variable stiffness composite cylinder uncertainty analysis by using reanalysis assisted Copula function
}

\author{
Zeng Yang ${ }^{1,3}$, Enying $\mathrm{Li}^{2}$, Hu Wang ${ }^{1,3, a}$ \\ ${ }^{1}$ State Key Laboratory of Advanced Design and Manufacturing for Vehicle Body, Hunan University, Changsha 410082, PR China \\ ${ }_{3}^{2}$ School of Logistics, Central South University of Forestry and Teleology, Changsha, 41004, P.R. China \\ ${ }^{3}$ Joint Centre for Intelligent New Energy Vehicle, Shanghai, China
}

\begin{abstract}
There are lots of uncertainties in variable-stiffness composite materials such as material properties, fibre volume fraction, geometries at various scale and matrix porosity. Commonly, these uncertainties are not always mutually independent and there exist correlations among these random input variables. These correlations may affect the output of composite significantly. To address these correlations, a novel approach for uncertainty analysis based on copula function assisted by reanalysis method is suggested. The Copula function is utilized to address the correlations of random input variables. Monte Carlo simulation (MCS) is employed to obtain the uncertainty analysis. Therefore, a large number of samples should be generated and the expensive computational cost is not feasible when the popular finite element (FE) model is utilized. To save the computational cost and make the uncertainty analysis feasible in practice, an efficient fast computation method, reanalysis method is integrated in the frame. The numerical test demonstrates that the proposed approach is an efficient uncertainty analysis tool for the practical engineering problems.
\end{abstract}

\section{Submitting the manuscript}

Variable stiffness laminates can improve material performance considerably and simultaneously keep the geometry size unchanged. Recently, there are a series of studies to develop these structures $[1,2]$. However, uncertainties such as material properties, fibre volume fraction, geometries at various scales, matrix porosity are still a challenge in real engineering [1]. Furthermore, these uncertainties are always not mutually independent and there exist correlations among the random input variables. These correlations may affect the output of the composite structure significantly. Fortunately, there exists a mathematical model - copula function $[3,4]$ which can address the correlations effectively. In this study, a D-vine copula [5] function is utilized to address the multivariate correlations among the random input variables.

In this work, Monte Carlo simulation (MCS) is utilized to propagate the uncertainty to the output of the structure. However, in this method, the accuracy of results mainly depends on the number of the samples. In other words, in order to obtain accurate results, it needs a large number of samples. These samples are commonly evaluated by FE simulations and the computational cost is too expensive to work. In order to address the timeconsuming problem, a fast and accurate numerical tool, named reanalysis method [10] is applied. We hope to use

\footnotetext{
a Corresponding author: wanghu@hnu.edu.cn
}

reanalysis make the simulation-based uncertainty analysis feasible.

The paper is organized as follows: Section 2 presents optimized Variable-stiffness plate. In Section 3, the detail of copula function is presented. Section 4 presents the fundamental theory of reanalysis. The results are discussed in Section 5.

\section{Optimized Variable stiffness plate}

In this study, the investigation is based on an optimized composite square plate [6] as shown in Fig.1. Suppose that there are 4 fibre plies and each ply's thickness is 0.5 . The material properties are referred from Ref.[7] and given in Table.1. The nodes on the left side are fixed and on the nodes on the right side are stretched. The forces are identical with the total value 200. Since the symmetrical (about $x$ ) structure and boundaries, the fibre paths are also symmetrical about axis x. Thus, relationship of path function of plies can be designed as: $g_{2}(x, y)=g_{1}(x,-y)$ and $g_{4}(x, y)=g_{3}(x,-y)$ (subs cripts represent the number of plies). The fibre paths are supposed to be a quadratic function of coordinate and given as

$$
g(x, y, \mathbf{a})=x+a_{1} y+a_{2} x y+a_{3} x^{2}+a_{4} y^{2}
$$

According to Ref.[6], the fibre orientation angle at $\left(x_{0}, y_{0}\right)$ can be given as 


$$
\theta(x, y)=\left\{\begin{array}{c}
\arctan \left(-\frac{1+a_{2} y+2 a_{3} x}{a_{1}+a_{2} x+2 a_{4} y}\right), a_{1}+a_{2} x+2 a_{4} y \neq 0 \\
\frac{\pi}{2}, a_{1}+a_{2} x+2 a_{4} y \neq 0
\end{array}\right.
$$

In this study, the fibre orientation angle is supposed to be a constant in an element and calculated by the centroid of the element. The detailed process of optimization is referred to Ref.[6] and one of the Pareto solution of hole plate is presented in Table. 2 and corresponding fibre path in each ply are illustrated in Fig.2.

However, in real engineering desgin, the ideal optimized fibre orientation angle is diffcult to be manufactured. There are always some deviations in the optimized fibre orientation angle. Since the manufacturing environment is the same, correlations will exist between the deviations in different ply in one plate. In this study, the deviations of fibre orientation angle in manufacturing are regarded as uncertainties. The details of the setting can be found in Section 3.

Table 1. Material parameters.

\begin{tabular}{|c|c|c|c|c|c|c|}
\hline $\begin{array}{c}\text { Material } \\
\text { parameter }\end{array}$ & $E_{L}$ & $E_{T}$ & $\lambda_{L T}$ & $G_{L T}$ & $G_{T N}$ & $G_{L N}$ \\
\hline Value (Mpa) & 137.9 & 10.34 & 0.29 & 6.89 & 3.9 & 6.89 \\
\hline
\end{tabular}

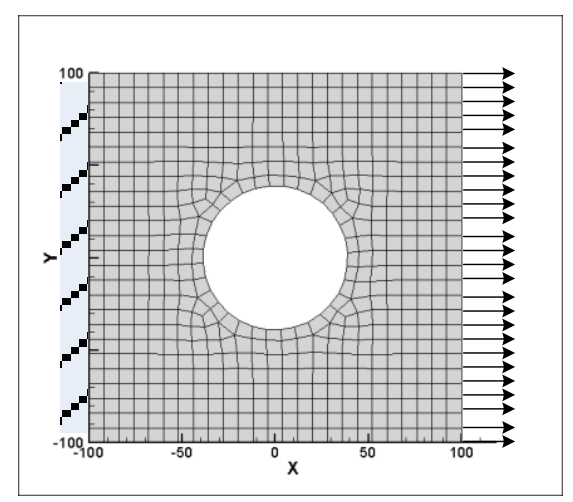

Figure 1. Square plate.

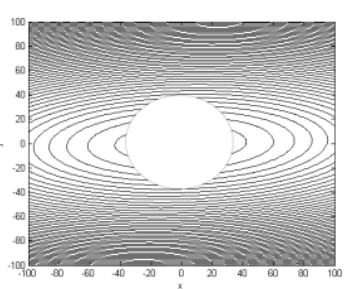

Ply 1

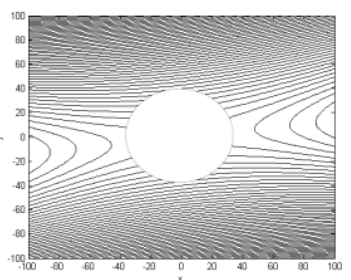

Ply 3

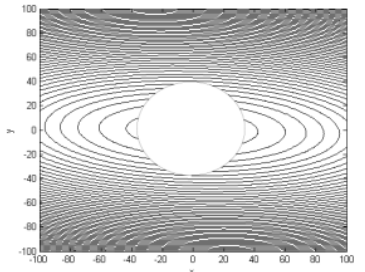

Ply 2

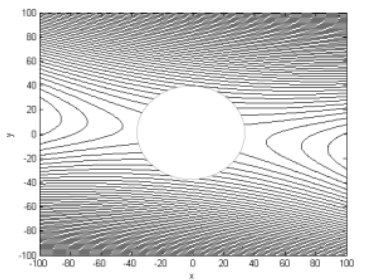

Ply 4
Figure 2. Fibre paths of given solution
Table 2. Optimal solution

\begin{tabular}{|c|c|c|c|c|c|}
\hline $\begin{array}{c}\text { Pareto } \\
\text { solutions }\end{array}$ & Plies & $a_{1}$ & $a_{2}$ & $a_{3}$ & $a_{4}$ \\
\hline \multirow{4}{*}{ solutions } & Ply 1 & -0.231 & -0.424 & 0.846 & 6.613 \\
\cline { 2 - 6 } & Ply 2 & 0.231 & 0.424 & 0.846 & 6.613 \\
\cline { 2 - 6 } & Ply 3 & 2.803 & -2.102 & -0.558 & 7.363 \\
\cline { 2 - 6 } & Ply 4 & -2.803 & 2.102 & -0.558 & 7.363 \\
\hline
\end{tabular}

\section{Copula function}

Copula function is a mathematical model which can estimate the connection between the marginal CDFs and joint CDF of $d$-dimensional multivariate distribution. In Sklar's theorem [3], if the marginal CDFs are continuous, the multivariate CDF can be determined by the copula uniquely. The expression is

$$
F\left(x_{1}, \ldots, x_{d}\right)=C\left(F_{1}\left(x_{1}\right), \ldots, F_{d}\left(x_{d}\right)\right)
$$

where $F_{i}\left(x_{i}\right)(i=1, \ldots d)$ is the $i$ th continuous marginal $\mathrm{CDF}$ of the multivariate distribution, and $C\left(F_{1}\left(x_{1}\right), \ldots, F_{d}\left(x_{d}\right)\right)$ represents the copula function. According to the relationship between PDF and CDF, the joint density function of multivariate distribution can be given as

$$
f\left(x_{1}, \ldots, x_{d}\right)=c\left(F_{1}\left(x_{1}\right) \ldots F_{d}\left(x_{d}\right)\right) f_{1}\left(x_{1}\right) . . f_{d}\left(x_{d}\right)
$$

where $c\left(F_{1}\left(x_{1}\right) \ldots F_{d}\left(x_{d}\right)\right)$ is defined as the density function of $C\left(F_{1}\left(x_{1}\right), \ldots, F_{d}\left(x_{d}\right)\right)$ and given as

$$
c\left(F_{1}\left(x_{1}\right) \ldots F_{d}\left(x_{d}\right)\right)=\frac{\partial C\left(F_{1}\left(x_{1}\right) \ldots F_{d}\left(x_{d}\right)\right)}{\partial F_{1} \ldots \partial F_{d}}
$$

Nevertheless, when $d$ is large, an ideal $C\left(F_{1}\left(x_{1}\right), \ldots, F_{d}\left(x_{d}\right)\right)$ is difficult to be constructed directly. Commonly, most bivariate copula function can fit the bivariate data well. Therefore, the pair-copula constructions (PCC) [5] are considered in the application of multivariate copulas. In this study, a D-vine copula based on PCC is used. Suppose that the $U=\left(U_{1}, \ldots, U_{d}\right)^{\prime}$ is a random vector in which the $U_{i}, i=1, \ldots, d$ represents marginal CDFs with uniformly $U(0,1)$. Suppose that $p$ and $q$ are integers which satisfy $(1 \leq p \leq q \leq d)$ and the symbol $p: q$ represents all integers ranging from $p$ to $q$, namely $p: q:=\{p, \ldots, q\}$. Define that $U_{p: q}$ represents the variables $\left\{U_{p}, \ldots, U_{q}\right\}$. According to a recursive decomposition, the $d$-dimensional density $f$ can be written as

$$
f\left(x_{1}, \ldots, x_{d}\right)=f\left(x_{1}\right) \prod_{k=2}^{d} f\left(x_{k} \mid x_{1}, \ldots, x_{k-1}\right)
$$

As to the copula density $c$, we have

$$
c\left(u_{1}, \ldots, u_{d}\right)=1 \prod_{k=2}^{d} c_{k \mid 1:[k-1]}\left(u_{k} \mid u_{1:[k-1]}\right)
$$

where 1 represents the marginal density of $U_{1}$. 
According to Ref.[5], defining $i[i+1] \mid \phi:=i[i+1]$, we have

$$
\begin{aligned}
& c\left(u_{1}, \ldots, u_{d}\right)=\prod_{j=1}^{d-1} \prod_{i=1}^{d-j} c_{i[i+j][i+1]:[i+j-1]}(\mathbf{u}, \mathbf{v}) \\
& \mathbf{u}=u_{i[i+1]:[i+j-1]} \\
& \mathbf{v}=u_{i+j[i[+1]:[i+j-1]}
\end{aligned}
$$

Equation (8) provides a D-vine copula which is factorized as the product of pair-copulas as shown in Fig.3.
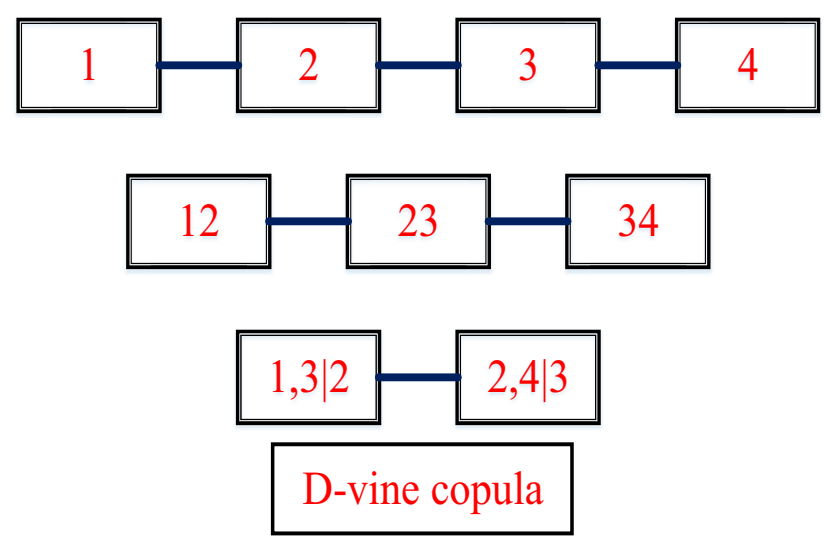

Figure 3. D-vine copula.

Commonly, the D-vine copula can be identified based on test samples via Akaike information criterion(AIC), goodness of fit (GOF) and Bayesian method $[5,8]$. In this study, in order to address the effect of correlations on the uncertainty analysis qualitatively, the correlations and distributions of the deviations are given. Suppose that the deviations of fibre orientation angle in a ply keep the same. Different plies subject to the same normal distribution with mean $\mu=0$ and standard deviation $\sigma=0.02$. This means that the deviations of fibre orientation is unbiased and only a relative small fluctuation. The correlations exist in different deviations from different plies. In order to generate the sample, suppose that all pair copulas are Frank. Since the aforementioned :

$$
g_{2}(x, y)=g_{1}(x,-y) \text { and } g_{4}(x, y)=g_{3}(x,-y)
$$

it's reasonable to assume that there is a negative correlation between ply 1 and ply 2 with a Kendall's tau $t a u=-0.7$ as shown in Fig.4a. So is the correlation of ply 3 and ply 4 . The Kendall's tau of the remaining correlations is supposed to be a small positive value with $\operatorname{tau}=0.3$ as shown in Fig. $4 \mathrm{~b}$. MC simulation is used to uncertainty analysis based these assumption and Dvine copula function. Through calculation, it is proved that $20000 \mathrm{MC}$ samples of these deviations which are drawn via Rosenblatt transformation [9] are enough to obtain the accurate results.

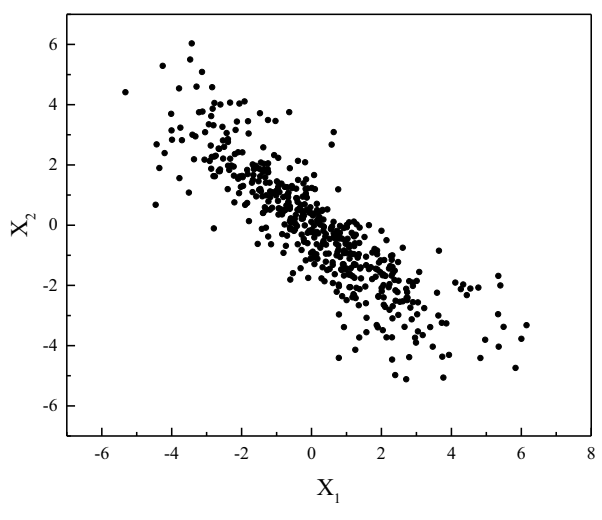

(a) tau $=-0.7$

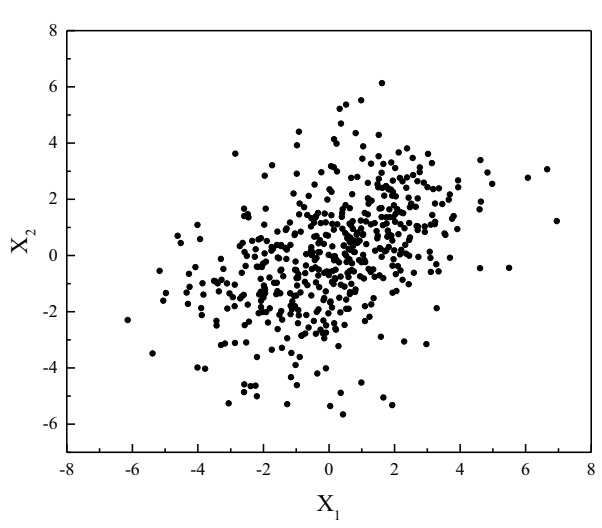

(b) $\quad$ tau $=0.3$

Figure 4. Sample distributions with different tau

\section{Reanalysis Method for uncertainty analysis}

Some of uncertainties, such as the deviations of $\theta(x, y)$ in this study, are involved in the variation of the stiffness matrix. The reanalysis is a powerful method to address this variation of stiffness matrix. Among approximate reanalysis methods, the CA [10] can simultaneously satisfy the require of accuracy and efficiency. Therefore, the CA is introduced in this study.

Regarding the optimized path function in Section 2 as the initial fibre orientation, and the equilibrium equation is

$$
\mathbf{K}_{0} \mathbf{r}_{0}=\mathbf{R}
$$

where $\mathbf{K}_{0}=\mathbf{K}(\theta(x, y))$ is the stiffness matrix, $\mathbf{R}$ is the load vector, and $\mathbf{r}_{0}$ is the initial displacement and can be solved as

$$
\mathbf{r}_{0}=\mathbf{K}_{0}^{-1} \mathbf{R}
$$

When there is uncertainty from the fibre orientation angle $\theta(x, y)$, the new equilibrium equation can be written as 


$$
\mathbf{K r}=\mathbf{R}
$$

Suppose

$$
\Delta \mathbf{K}=\mathbf{K}-\mathbf{K}_{0}
$$

Equation(11) can be written as

$$
(\mathbf{K}+\Delta \mathbf{K}) \mathbf{r}=\mathbf{R}
$$

Suppose that the displacement is a linear combination of a series of basis vectors as

$$
\mathbf{r}=y_{1} \mathbf{v}_{1}+y_{2} \mathbf{v}_{2}+\cdots+y_{s} \mathbf{v}_{s}=\mathbf{r}_{B} \mathbf{y}
$$

where $\quad \mathbf{v}_{i}(i=1,2, \ldots s)$ is basis vector and $y_{i}(i=1,2, \ldots, s)$ is corresponding coefficients, $s$ represents the number of basis vectors

$$
\begin{aligned}
& \mathbf{r}_{B}=\left[\begin{array}{llll}
\mathbf{v}_{1} & \mathbf{v}_{2} & \cdots & \mathbf{v}_{s}
\end{array}\right] \\
& \mathbf{y}=\left[\begin{array}{llll}
y_{1} & y_{2} & \cdots & y_{s}
\end{array}\right]^{T}
\end{aligned}
$$

According to Ref.[10], we can construct the basis vectors as

$$
\begin{aligned}
& \mathbf{v}_{1}=\mathbf{r}_{0} \\
& \mathbf{v}_{i}=-\mathbf{B} \mathbf{v}_{i-1}, i=2,3, \ldots, s \\
& \mathbf{B}=\mathbf{K}_{0}^{-1} \Delta \mathbf{K}
\end{aligned}
$$

Substituting Eq.(14) into Eq.(11), then premultiplying both side with $\mathbf{r}_{B}{ }^{T}$, we have

$$
\mathbf{K}_{r} \mathbf{y}=\mathbf{R}_{r}
$$

where

$$
\begin{aligned}
& \mathbf{K}_{r}=\mathbf{r}_{B}{ }^{T} \mathbf{K} \mathbf{r}_{B} \\
& \mathbf{R}_{r}=\mathbf{r}_{B}{ }^{T} \mathbf{R}
\end{aligned}
$$

$\mathbf{y}$ can be obtained via solving Eq.(17). Thus, the approximation of $\mathbf{r}$ can be obtained via substituting $\mathbf{y}$ into Eq.(14)

In uncertainty analysis of variable-stiffness laminate, the optimized solution can be regard as the initial condition which can be solved by using full analysis according to Eq.(10). Then all samples of uncertainties can be added to the stiffness matrix and then solved by using the CA method. Since the scale of $\mathbf{K}$ is much larger than $\mathbf{K}_{r}$, the computational cost can be reduced significantly.

\section{Results and discussions}

The D-vine copula can address the correlation effectively. It does not need joint PDF of the random input variables. It only depends on the test samples. With the test samples, the pair-copula are quite easy to be constructed. Based on the identified vine copula, 20000 MC samples are generated to simulate the joint PDF in this study. Thus, the correlations exist in the samples and can propagate to results completely.
Commonly, some of the uncertainties are involved in the variation of part of stiffness matrix. Since the purpose of the reanalysis method is to analyze based on the changed part of stiffness matrix $\mathbf{K}_{r}$ which is much smaller than the $\mathbf{K}$, it presents a powerful performance in this kind of uncertainty analysis. With the full FE analysis, it takes 21 seconds for each evalutions. While with reanalysis method, it only takes less than 2 seconds. The reanalysis method greatly improves the efficiency of computation. 8 test samples are generated to test the accuracy of the reanalysis and the results are presented in Table.3. It is shown that the results of reanalysis are almost equal to the results of full analysis. The reanalysis is quite accurate.

Table. 3. The results of the test samples

\begin{tabular}{|c|c|c|c|c|}
\hline \multirow{2}{*}{} & \multicolumn{4}{|c|}{ The maximum displacements $\left(10^{-6}\right)$} \\
\cline { 2 - 5 } & Full analysis & \multicolumn{2}{c|}{ Reanalysis } \\
\hline Numbers & $\mathrm{X}$ & $\mathrm{Y}$ & $\mathrm{X}$ & $\mathrm{Y}$ \\
\hline 1 & 4.7070 & 1.2880 & 4.7069 & 1.2880 \\
\hline 2 & 4.6967 & 1.2434 & 4.6967 & 1.2434 \\
\hline 3 & 4.6296 & 1.1719 & 4.6296 & 1.1719 \\
\hline 4 & 4.6102 & 1.2374 & 4.6102 & 1.2374 \\
\hline 5 & 4.6173 & 1.2088 & 4.6173 & 1.2088 \\
\hline 6 & 4.6197 & 1.1670 & 4.6197 & 1.1670 \\
\hline 7 & 4.8146 & 1.3177 & 4.8146 & 1.3177 \\
\hline 8 & 4.6577 & 1.1787 & 4.6576 & 1.1787 \\
\hline
\end{tabular}

In the optimized variable-stiffness plate, the maximum displacement of $X$ direction is $4.629 \times 10^{-6}$ and the maximum displacement of $Y$ direction is $1.209 \times 10^{-6}$. When the uncertainties are taken account, the displacements of the results are shown in Fig.5. The means of the corresponding maximum displacements are $4.634 \times 10^{-6}$ and $1.211 \times 10^{-6}$, respectively. As for maximum displacement of $\mathrm{X}$ direction, the minimum value is $4.436 \times 10^{-6}$ and the maximum value is $4.890 \times 10^{-6}$. This means that the uncertainty of maximum displacement of $\mathrm{X}$ direction may reach to $10 \%((4.890-4.436) / 4.629=9.81 \%)$. For maximum displa-cement of $\mathrm{Y}$ direction, the minimu$\mathrm{m}$ value is $1.041 \times 10^{-6}$ and maximum value is $1.380 \times 10^{-6}$. The uncertainty of maximum displacement of $\mathrm{Y}$ direction is $28.04 \%$. Either $9.81 \%$ or $28.04 \%$ is a quite large value of uncertainty in the real engineering problems since the deviations of the fibre orientation angle is quite small $(\sigma=0.02)$. When in Variablestiffness plate design, taking the uncertainties into account is quite important to design a reliable structure. 

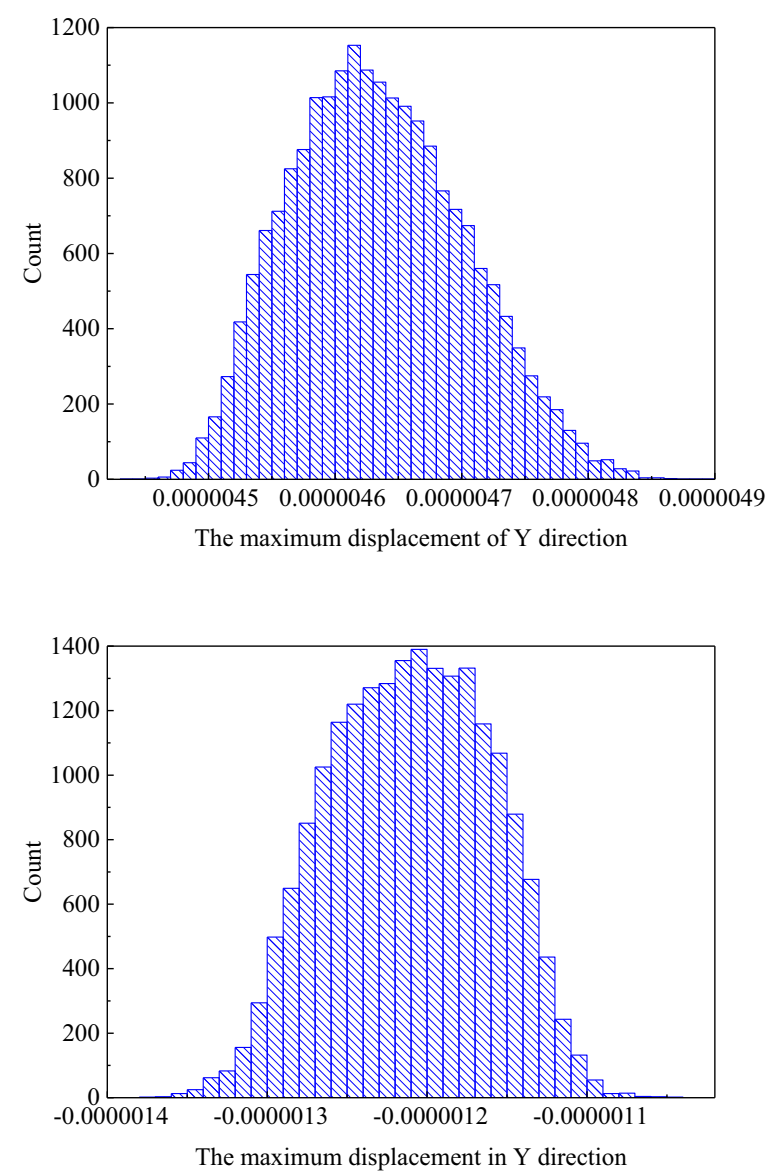

Figure 5. The maximum displacements with uncertainty

\section{Remarks}

- We present a novel approach for uncertainty analysis based on copula function assisted by reanalysis method.

- The correlations among random input variables are characterized as a D-vine copula function. In this way, the uncertainty analysis taking all kinds of factors of random input variables into account can be implemented conveniently.

- Reanalysis method is integrated in the frame to save the computational cost and make the uncertainty analysis feasible in practice.

\section{Acknowledgements}

This work has been supported by Project of the Program of National Natural Science Foundation of China under the Grant Numbers 11172097, 11302266 and 61232014.

\section{References}

1. Fayazbakhsh K, Nik M A, Pasini D, et al. Defect layer method to capture effect of gaps and overlaps in variable stiffness laminates made by automated fibre placement. Composite Structures, 2013, 97: 245-251.
2. Rouhi M, Ghayoor H, Hoa S V, et al. Effect of structural parameters on design of variable-stiffness composite cylinders made by fibre steering. Composite Structures, 2014, 118: 472-481.

3. Sklar, A. Fonctions de répartition à $\mathrm{n}$ dimensions et leurs marges. Publications de 1'Institut de Statistique de l'Université de Paris 8, 1959: 229-231.

4. Nelsen, R B. An Introduction to Copulas, Springer, 1999: New York.

5. Min A, Czado C. Bayesian model selection for Dvine pair-copula constructions. Canadian Journal of Statistics, 2011, 39(2): 239-258.

6. Guanxin H, H Wang, G.Y. Li. An Efficient Reanalysis for Variable-stiffness Composite Design by Using Path Function. Composite Strcutures. (Submitted).

7. Brampton CJ, Kim HA. Optimization of tow steered fibre orientation using the level set method. In: 10th World Congress on Structural and Multidisciplinary Optimization (WCSMO/10), Orlando, 19-24 May 2013.

8. Gruber L, Czado C. Sequential Bayesian model selection of regular vine copulas. Bayesian Analysis, 2015.

9. Rosenblatt, M. Remarks on a Multivariate Transformation. The Annals of Mathmatical Statistics, 1952; 23(3): 470-472.

10. Kirsch U, Moses F. An improved reanalysis method for grillage-type structures. Computers and Structures, 1998; 68:79-88. 